\title{
Neurological complications of anterior spinal surgery for kyphosis with normal somatosensory evoked potentials (SEPs)
}

\author{
L Pelosi, A Jardine, J K Webb
}

\begin{abstract}
We report a case of neurological complications of anterior release for correction of kyphosis. After the operation, the patient had pyramidal weakness and decreased pain sensation below $T 5$, whereas light touch, proprioception and vibration sensation were intact. Clinical and neurophysiological findings in this patient suggested a partial lesion of the spinal cord probably due to ischaemia in the territory of the anterior spinal artery. Intraoperative and postoperative tibial nerve SEPs remained normal, which stresses the need for recording from the motor pathways. (F Neurol Neurosurg Psychiatry 1999;66:662-664)
\end{abstract}

Keywords: spinal surgery; SEPs; motor deficits

We report a case of a postoperative partial spinal cord syndrome, affecting predominantly pyramidal and spino-thalamic pathways below T5, which complicated an anterior spinal release for kyphosis and was associated with normal SEPs.

\section{Case report}

Department of Clinical Neurophysiology L Pelosi

\section{Department of} Anaesthesia

A Jardine

Centre for Spinal Studies and Surgery, University Hospital, Queen's Medical Centre, Nottingham, UK

J K Webb

Correspondence to: Dr Luciana Pelosi, Department of Clinica Neurophysiology, University Hospital, Queen's Medical Centre, Nottingham NG7 $2 \mathrm{UH}, \mathrm{UK}$.

Received 8 May 1998 and in revised form

24 August 1998

Accepted 16 September 1998

The anterior release was carried out via a right thoracoscopic approach with SEP monitoring. Segmental vessels were ligated from T6 to T11. T6-7 to T10-11 discs were excised and bone grafts placed into disc spaces.

Following $150 \mu \mathrm{g}$ fentanyl, anaesthesia was induced with $200 \mathrm{mg}$ of propofol. Intubation was performed after paralysis with $10 \mathrm{mg}$ of vecuronium and anaesthesia maintained with $50 \%$ nitrous oxide/oxygen and isoflurane at $1.2 \%$ end tidal with supplemental narcotic and relaxant as required. Mechanical ventilation was adjusted to maintain end tidal carbon dioxide at $4.8 \%$ and mean arterial pressure (MAP) held between 75 and $80 \mathrm{~mm} \mathrm{Hg}(80 \%$ preoperative).

During suture of the thoracoscopic sites there was a transient change in the cerebral function monitoring (CFAM) with amplitude attenuation and dispersion lasting about 2-3 minutes. These changes were mirrored solely by a rise in MAP to $100 \mathrm{~mm} \mathrm{Hg}$ returning to 75 $\mathrm{mm} \mathrm{Hg}$ over 15 minutes. SEPs recorded during and after the CFAM change remained normal. The initial neurological examination after recovery of consciousness showed no detectable changes compared to the preoperative state. Some 40 minutes later there was a progressive deterioration of motor function in the lower limbs which commenced in the proximal muscles and lead to total paralysis. Toe movements returned partially after mannitol $0.5 \mathrm{~g} / \mathrm{kg}$, but neurological examination 2 hours after recovery of consciousness showed severe paraparesis worse proximally, absent abdominal reflexes, suspended analgesia between T5 and T11 on the left, hypalgesia at the same levels on the right and asymmetric loss of pain sensation in the legs (left worse than right). Light touch, proprioception and vibration sensation were intact. No changes of vital parameters occurred at the time when neurological deficits became manifest.

MRI of the spinal cord was normal.

A week after the operation, there was still an asymmetric pyramidal weakness of the legs worse on the right and reduced pain sensation below T5. There was a gradual improvement of motor function, but 4 weeks after surgery the patient was still disabled needing bilateral support to walk. Three months after the operation motor function had returned to normal.
SOMATOSENSORY EVOKED POTENTIALS (SEPS)

Preoperative and postoperative SEPs to stimulation of the tibial nerves at the ankle (squarewave electrical pulses of $0.1 \mathrm{msec}$ duration, $3 \mathrm{~Hz}$, intensity adjusted to produce a visible plantar flexion of the foot) were recorded via a pair of silver discs at $\mathrm{Cz}$ and $\mathrm{Fz}(10-20$ International System). Bandpass filters were $30-5000 \mathrm{~Hz}$ and analysis time $100 \mathrm{~ms}$. 
A

Left TN

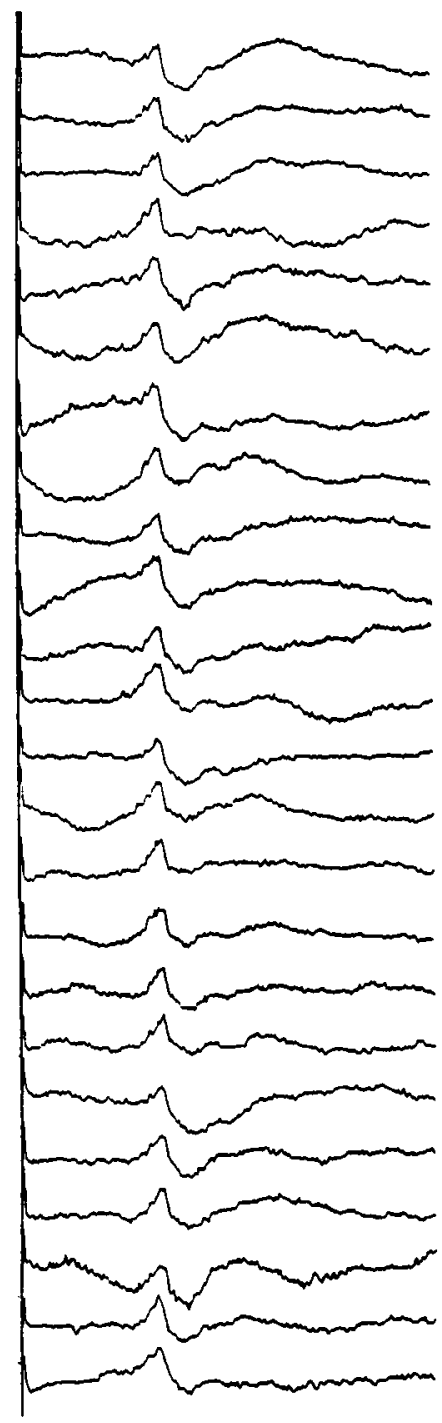

Right TN

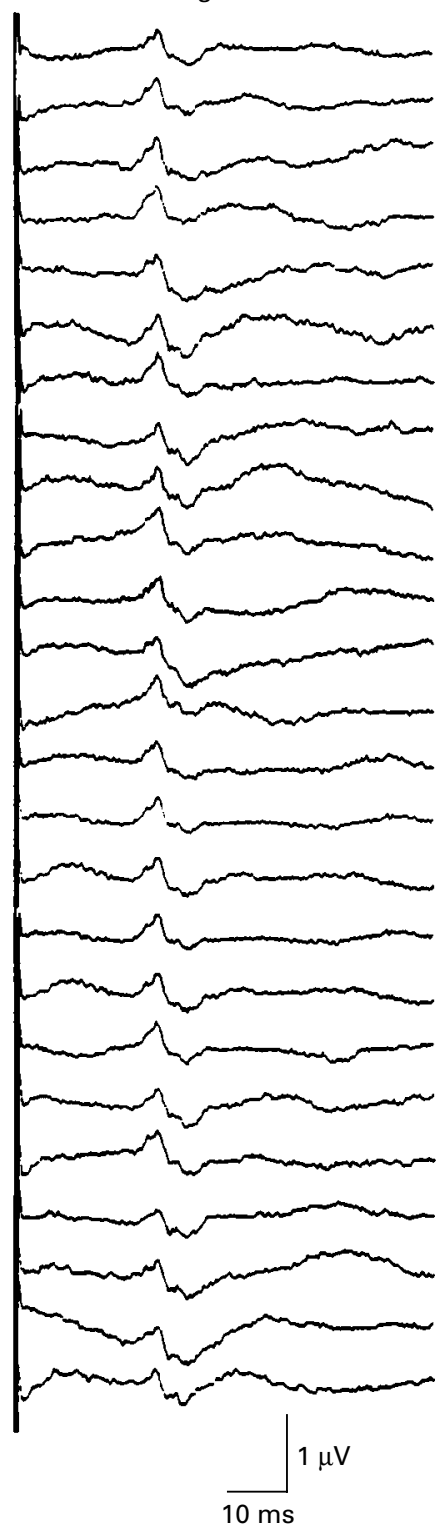

B

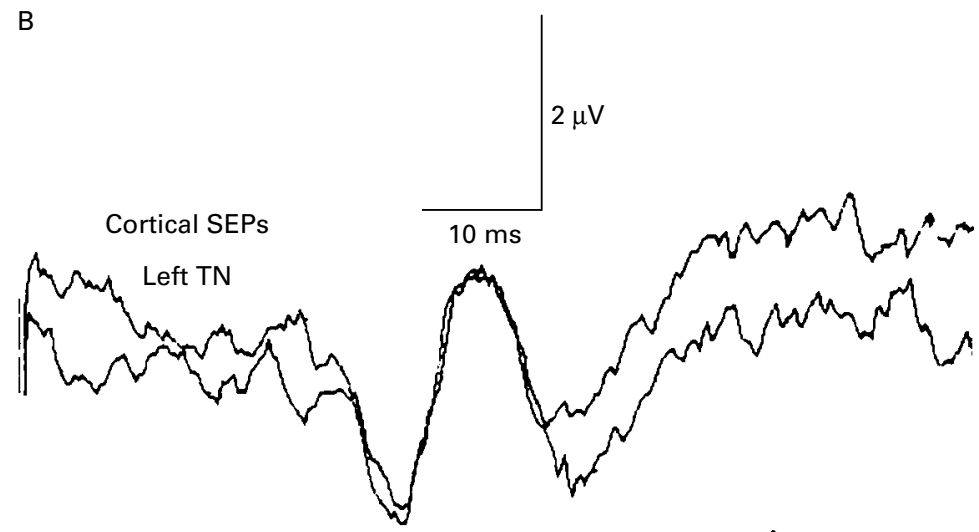

Intraoperative SEPs to alternate stimulation of the tibial nerves at the ankle $(7 \mathrm{~Hz}, 0.1 \mathrm{~ms}$ duration, intensity adjusted as above prior to administration of muscle relaxants) were derived from a cervical-to-scalp montage (the cathode over the $\mathrm{C} 2$ and $\mathrm{C} 5$ spinous processes and the anode at $\mathrm{Fz}$ ) using silver disc electrodes. Surface recordings of the peripheral nerve responses at the knee were obtained simultaneously to ensure adequacy of stimulation. SEPs were recorded continuously during the first two hours of the operation, following which monitoring was discontinued as no instrumentation and corrective manoeuvres potentially hazardous for the spinal cord were to be implemented. SEP monitoring was resumed at the end of the operation before closure.

Intraoperative SEPs remained normal and symmetric throughout the monitoring time. SEPs recorded at the end of the operation were normal with no significant change of amplitude and latency relative to the baseline responses (fig A). Both the preoperative SEPs and the SEPs recorded the day after the operation when the patient was severely disabled were normal (fig B).

\section{Discussion}

In our patient, thoracoscopic release for correction of kyphosis was complicated by a partial spinal cord lesion affecting pyramidal and spino-thalamic pathways below $\mathrm{T} 5$, but sparing posterior sensory cord functions. We could not document the time when the spinal cord damage occurred, because all vital parameters remained unchanged throughout the operation, except for a sudden decrease of the CFAM amplitude. We could identify no reason for this change, but it is possible that damage to the spinal cord might have begun at that time. Immediate postoperative examination was apparently normal, suggesting that the pathological process evolved over a period of time. Again, no change of vital parameters occurred at the time when the neurological deficits became manifest. Three delayed neurological complications following spinal surgery, two of which purely motor, have been mentioned but not further discussed, in a previous paper. ${ }^{1}$ Delayed postoperative paraplegia has also been reported in a 46 year old woman following revision of scoliosis surgery. ${ }^{2}$ Neurological deficits begun three and a half hours after skin closure and coincided with a fall in blood pressure probably caused by hypovolaemia. Interestingly, in the same case report, introperative falls of blood pressure to the same value blamed postoperatively for cord malfunction were not associated with SEP change.

(A) Sequential recordings of intraoperative SEPS to alternate stimulation of the left and right tibial nerves at the ankle. Surface recording from $C 2$ (2nd cervical spinous process) $-F z$. Note that SEPs recorded at the end of the operation (last two traces on each side) were normal. (B) Postoperative cortical SEPS $(C z-F z)$ to stimulation of the tibial nerves $(T N)$. The patient had severe motor deficits at the time when these SEPs were recorded. 
The distribution and type of neurological deficit in our patient suggest a vascular mechanism. Ischaemia of the anterior spinal artery at thoracic level would be consistent with a partial lesion affecting the anterolateral and central cord. The posterior third of the cord, which has an independent vascularisation via the posterior spinal arteries, would be expected to be spared. In the thoracic region, the anterior cord is particularly vulnerable to ischaemia, because of the narrowing of the anterior spinal artery and the relative paucity of anastomotic radicular branches. The anterior spinal artery syndrome is a complication of surgery on the thoracic and abdominal aorta and has been reported as a rare complication of posterior procedures for scoliosis and, in one instance, following anterior spinal fusion for scoliosis. ${ }^{3-5}$ It is possible that other case reports of predominantly motor postoperative sequelae with preserved intraoperative SEPs were also due to a vascular mechanism. ${ }^{1}$ As the sensory pathways which generate the SEPs traverse predominantly the posterior cord, it is not surprising that SEPs, both intraoperative and postoperative, remained normal in our patient. There is the possibility that we have missed a transient change of the SEPs which might have occurred during the period of time when monitoring was discontinued. However, normal SEPs were recorded both at the end of the operation and postoperatively when the patient had severe motor deficits.

Recording from the motor pathways should be expected to offer a better chance of detecting and, hopefully, preventing motor deficits due to anterior spinal artery syndrome. Furthermore, studies in non-human primates have shown that the corticospinal pathways are far more sensitive to ischaemia than the sensory tracts. ${ }^{67}$ For moderate degrees of ischaemia, profound attenuation of the motor responses could occur without changes in the SEPs. ${ }^{6}$ In the case report by Ginsburg and colleagues, the patient awoke paraplegic from posterior instrumentation despite normal intraoperative SEPs. Changes of the SEPs became manifest hours later. ${ }^{8}$ Methods for intraoperative assessment of the spinal motor pathways have been developed and improved in recent years, but various technical difficulties have prevented their widespread use. ${ }^{9-12}$ However, the case we report clearly demonstrates that major motor disability can be associated with entirely normal SEPs. Thus, SEPs cannot be relied upon to detect motor dysfunction.

Neurophysiological monitoring is generally performed during posterior instrumentation in order to prevent damage to the cord from corrective manoeuvres. However, an ischaemic lesion can also occur independently at any stage in both the anterior and posterior procedures. This case stresses the importance of monitoring motor function during anterior procedures.

1 Lesser RP, Raudzens P, Luders $\mathrm{H}$, et al. Postoperative neurological deficits may occur despite unchanged intraoperative somatosensory evoked potentials. Ann Neurol 1986;19:22-5.

2 Taylor BA, Webb PJ, Hetreed M, et al. Delayed postoperative paraplegia with hypotension in adult revision scoliosis surgery. Spine 1994;19:470-4.

3 Daube JR. Intraoperative monitoring by evoked potentials for spinal cord surgery: the pros. Electroencephalogr Clin for spinal cord surgery: the

4 Szilagyi DE, Hageman JH, Smith RF, et al. Spinal cord damage in surgery of the abdominal aorta. Surgery 1978;83:38-56.

5 Ben-David B, Haller G, Taylor P. Anterior spinal fusion complicated by paraplegia. A case report of a false-negative somatosensory-evoked potential. Spine 1987;12:536-9.

6 Bennett M H. Effects of compression and ischemia on spinal cord evoked potentials. Exp Neurol 1983;80:508-19.

7 Owen JH, Naito M, Bridwell $\mathrm{KH}$, et al. Relationship between duration of spinal cord ischemia and postoperative neurologic deficits in animals. Spine 1990;15:847-51.

8 Ginsburg HH, Shetter AG, Raudzens PA. Postoperative paraplegia with preserved intraoperative somatosensory paraplegia with preserved intraoperative som

9 Boyd SG, Rothwell JC, Cowan JMA, et al. A method of monitoring function in corticospinal pathways during scolmonitoring function in corticospinal pathways during scolNeurol Neurosurg Psychiatry 1986;49:251-57.

10 Burke D, Hicks R, Stephen J, et al. Assessment of corticospinal and somatosensory conduction simultaneously during scoliosis surgery. Electroencephalogr Clin Neurophysiol 1992;85:388-96.

11 Jones SJ, Harrison R, Koh KF, et al. Motor evoked potential monitoring during spinal surgery: responses of distal limb muscles to transcranial cortical stimulation with pulse trains. Electroencephalogr Clin Neurophysiol 1996;100:375-83.

12 Pelosi L, Caruso G, Balbi P. Characteristics of spinal potentials to transcranial motor cortex stimulation: Intraoperative recording. In: Rossini P, Marsden CD, eds. Noninvasive stimulation of brain and spinal cord. Fundamentals and clinical applications. Alan R Liss 1988:297-4. 